\title{
2. Finanzströme
}

\section{Gérard Perroulaz and Christoph Stamm}

\section{(2) OpenEdition}

\section{Journals}

Electronic version

URL: http://journals.openedition.org/sjep/240

DOI: $10.4000 /$ sjep.240

ISSN: 1663-9677

\section{Publisher}

Institut de hautes études internationales et du développement

\section{Printed version}

Date of publication: 1 avril 2004

Number of pages: $269-287$

ISSN: $1660-5926$

\section{Electronic reference}

Gérard Perroulaz und Christoph Stamm, « 2. Finanzströme», Schweizerisches Jahrbuch für

Entwicklungspolitik [Online], 23-1 | 2004, Online erschienen am: 30 April 2010, abgerufen am 08 September 2020. URL : http://journals.openedition.org/sjep/240 ; DOI : https://doi.org/10.4000/sjep. 240

(c) The Graduate Institute 


\section{Finanzströme}

\section{Kommentar}

Der Entwicklungshilfeausschuss der OECD (DAC) gibt statistische Daten über sämtliche finanzielle Beiträge an die Entwicklungsländer heraus. Dabei unterscheidet das DAC vier Arten von Finanzströmen: öffentliche Entwicklungshilfe, andere Beiträge des öffentlichen Sektors (darunter die staatlichen Exportkredite), Geschenke privater Hilfsorganisationen und private Beiträge zu Marktbedingungen (Privatkapitalströme). Die privaten Mittel setzen sich aus Direktinvestitionen, Wertpapieranlagen und privaten Exportkrediten zusammen. Nach Ansicht der Schweiz sind die statistischen Angaben betreffend die privaten Kapitalströme sehr lückenhaft. Die Schweizerische Nationalbank veröffentlicht Angaben über die ausländischen Direktinvestitionen (Tabellen 2.4), jedoch sind die Angaben über die Wertpapieranlagen bei weitem nicht vollständig.

\section{Grafik 3 : Finanzbeiträge an die Entwicklungsländer 1994-2002 (Mio Fr.)}

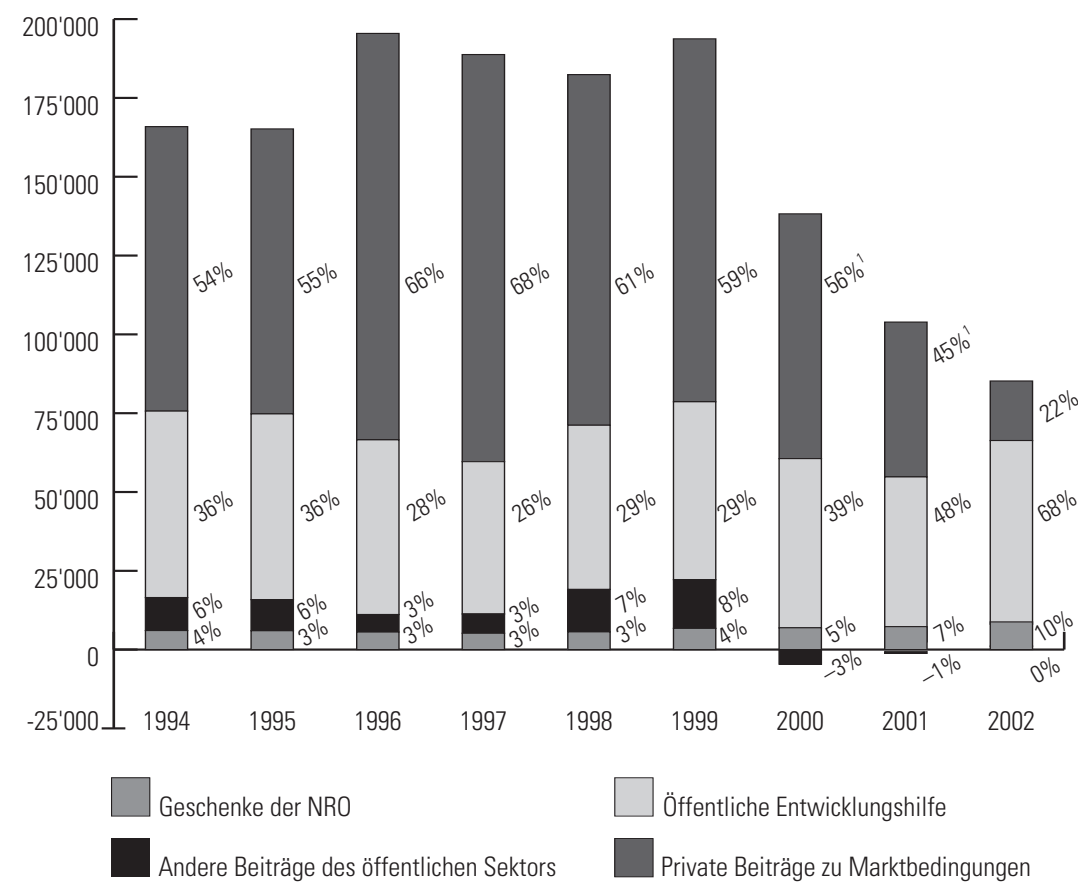

Quellen: OECD, Coopération pour le développement, Berichte 2002 (Tabelle 14), 1999 (Tabelle 14) und 1997 (Tabelle 21).

1 Die Prozentwerte beruhen auf dem Gesamtbetrag der positiven Flüsse. 
Grafik 3 zeigt die Entwicklung der finanziellen Beiträge an die Entwicklungsländer seit 1994 auf. Die Beiträge des Privatsektors hatten bis 1997 an Bedeutung zugenommen, wogegen die öffentliche Entwicklungshilfe im Rückgang begriffen war. Ab dem Jahr 2000 stellt man eine deutliche Trendwende fest, da die Privatkapitalströme im freien Fall begriffen sind und von 115 Milliarden Dollar 1999 auf 19 Milliarden Dollar 2002 absinken. Die öffentliche Entwicklungshilfe ist ihrerseits viel weniger volatil als die privaten Kapitalströme. Ihr relativer Anteil ist allerdings seit dem historischen Tiefstand von 26 Prozent im Jahr 1997 auf 68 Prozent 2002 angestiegen. Die „anderen Beiträge des öffentlichen Sektors“ erwiesen sich in den letzten Jahren als negativ, da die Rückzahlungen höher als die gewährten Kredite waren.

Vergleicht man die Beiträge der verschiedenen DAC-Mitgliedsländer, so kann man feststellen, dass zwischen dem Bruttovolkseinkommen (BVE) pro Einwohner, dem relativen Umfang der Privatkapitalströme und dem Umfang der öffentlichen Entwicklungshilfe keine Korrelation besteht (Tabelle 2.2). In der Einführung zum 3. Teil der Statistiken führen die Grafiken 9 und 10 die Einstufung der Länder gemäss den öffentlichen Entwicklungshilfeaufwendungen (in Milliarden Dollar und in Prozent des BVE) auf.

\section{Auslandsguthaben und -verpflichtungen der in der Schweiz niedergelassenen Banken (Grafik 4 und Tabellen 2.3.B)}

Die Schweizerische Nationalbank (SNB) gibt eine Statistik der Auslandsguthaben und -verpflichtungen der in der Schweiz niedergelassenen Banken heraus. Die Angaben beruhen auf den Informationen von 110 im Auslandsgeschäft tätigen Banken. Die Guthaben und Verpflichtungen der Niederlassungen (nicht aber der Tochtergesellschaften) im Ausland werden in der Statistik erfasst. Die Schweizerische Nationalbank unterscheidet die von den Banken auf eigene Rechnung getätigten (in der Bilanz aufgeführten) Geschäfte (Tabelle 2.3.B.1) von den auf Rechnung und Risiko der Kunden getätigten Transaktionen (Treuhandgeschäfte, Tabelle 2.3.B.2). Die nachstehende Grafik 4 führt die wichtigsten Herkunftsorte der Verpflichtungen der Schweiz gegenüber den Kunden aus Entwicklungsländern, aus den „fortgeschritteneren Entwicklungsländern“, den mittel und osteuropäischen Ländern und den Neuen Unabhängigen Staaten (MOEL/NUS) an. Die Schweizer Banken verwalteten somit Ende 2002 Mittel auf Rechung ausländischer Kunden in Höhe von insgesamt 267 Milliarden Franken, davon 80 Milliarden Franken für Kunden aus Entwicklungsländern (2. Spalte von Tabelle 2.3.B.2). Aus der Grafik 4 ist zu ersehen, dass die Schweizer Banken vor allem Mittel aus Steuerparadiesen und OffshoreFinanzzentren, aus relativ fortgeschrittenen Entwicklungsländern und Erdölausfuhrländern verwalten. Jedoch ist auch ein sehr armes Land (Liberia) aufgeführt sowie ein Land, das 2002 mit einer schweren Wirtschaftskrise zu kämpfen hatte (Argentinien). 


\section{Grafik 4 : Netto-Auslandsverpflichtungen der in der Schweiz niedergelassenen Banken (Treuhandgeschäfte), in Millionen Franken, Stand am 31. Dezember 2002}



Quelle: Schweizerische Nationalbank, Das Schweizerische Bankwesen 2002, 2003, <www.snb.ch>. 


\section{Schweizerische Direktinvestitionsbestände im Ausland}

(Grafik 5, Tabelle 2.4.A)

Grafik 5 macht die Konzentration der schweizerischen Direktinvestitionen in den Industrieländern deutlich. Unter den Entwicklungsländern ist Mittel- und Südamerika das bevorzugte Bestimmungsgebiet der Investitionen.

\section{Grafik 5 : Geografische Aufteilung der schweizerischen Direktinvestitions- bestände im Ausland, 2002 (Gesamtbetrag 409'655 Mio. Fr.)}

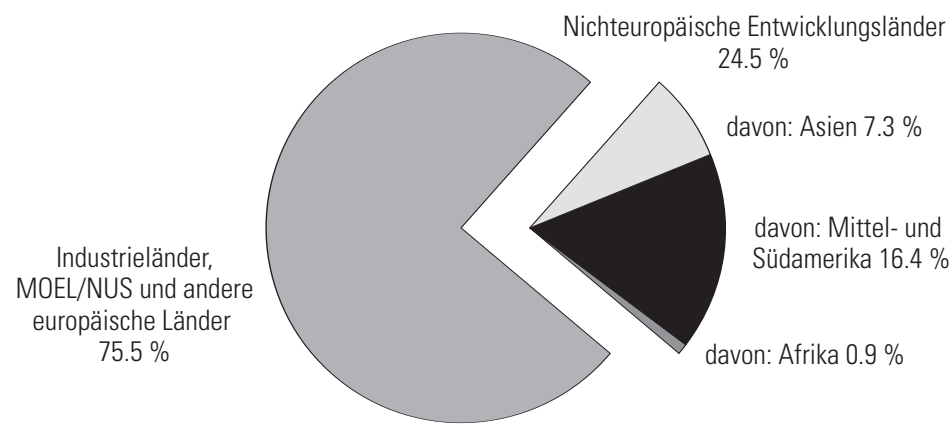

\section{Personalbestand in den schweizerischen Unternehmen im Ausland}

(Grafik 6, Tabelle 2.4.B)

Die oben beschriebene Tendenz widerspiegelt sich auch in der geografischen Aufteilung des Personals der schweizerischen Unternehmen. Eine Ausnahme hierzu bildet das in den asiatischen Entwicklungsländern beschäftigte Personal, das zahlenmässig stärker ist als das Personal in Mittel- und Südamerika.

\section{Grafik 6: Geografische Aufteilung des Personalbestandes der schweizerischen Unternehmen im Ausland, 2002 (Gesamtbestand 1'826'740 Personen)}






\subsection{Allgemeine Übersicht über die Finanzströme in die Entwicklungsländer}

A. Von der Schweiz angegebene Nettoflüsse (2000, 2001 und 2002)

\begin{tabular}{|c|c|c|c|c|c|c|}
\hline \multirow{2}{*}{ Kategorien } & 200 & 2001 & 2002 & 2000 & 2001 & 2002 \\
\hline & \multicolumn{3}{|c|}{ In Millionen Franken } & \multicolumn{3}{|c|}{ In Prozent des BVE } \\
\hline 1. Privatkapital ${ }^{1}$ & 1681,1 & $-2112,0$ & 1695,4 & 0,39 & $-0,47$ & 0,37 \\
\hline davon: Direktinvestitionen & 1914,1 & $-1867,4$ & 1902,4 & & & \\
\hline Exportkredite & 844,0 & $-242,9$ & $-207,1$ & & & \\
\hline Wertpapieranlagen & $-1076,9$ & $-1,7$ & - & & & \\
\hline 2. Öffentliche Entwicklungshilfe ${ }^{2}$ & 1502,2 & 1531,7 & 1461,8 & 0,34 & 0,34 & 0,32 \\
\hline 3. Sonstige öffentliche Beiträge ${ }^{3}$ & 13,5 & 10,1 & 4,7 & 0,00 & 0,00 & 0,00 \\
\hline $\begin{array}{l}\text { 4. Geschenke privater } \\
\text { Hilfsorganisationen }\end{array}$ & 274,1 & 303,4 & 314,5 & 0,06 & 0,07 & 0,07 \\
\hline Insgesamt & 3471,0 & $-266,8$ & 3476,3 & 0,80 & $-0,06$ & 0,75 \\
\hline \multicolumn{7}{|l|}{ davon : } \\
\hline zu Marktbedingungen $(=1+3)$ & 1694,7 & $-2101,9$ & 1700,0 & 0,3 & $-0,4$ & 0,3 \\
\hline zu liberalen Bedingungen $(=2+4)$ & 1776,4 & 1835,1 & 1776,3 & 0,4 & 0,4 & 0,3 \\
\hline BVE der Schweiz & 435478,2 & 450501,8 & 462369,6 & & & \\
\hline
\end{tabular}

Quelle: OECD, Statistical Annex of the 2003 Development Co-operation Report, Paris, Januar 2004.

\subsection{Allgemeine Übersicht über die Finanzströme in die Entwicklungsländer}

B. Von sämtlichen DAC-Mitgliedsländern angegebene Nettoflüsse (2000, 2001 und 2002)

\begin{tabular}{|c|c|c|c|c|c|c|}
\hline \multirow{2}{*}{ Kategorien } & 200 & 2001 & 2002 & 2000 & 2001 & 2002 \\
\hline & \multicolumn{3}{|c|}{ In Millionen Franken } & \multicolumn{3}{|c|}{ In Prozent des BVE } \\
\hline 1. Privatkapital ${ }^{1}$ & 78128,0 & 49745,0 & 18899,0 & 0,32 & 0,21 & 0,08 \\
\hline davon: Direktinvestitionen & 71729,0 & 66041,0 & 48844,0 & & & \\
\hline Exportkredite & 7352,0 & 2736,0 & 14,0 & & & \\
\hline Wertpapieranlagen & $-953,0$ & $-19032,0$ & $-29959,0$ & & & \\
\hline 2. Öffentliche Entwicklungshilfe ${ }^{2}$ & 53734,0 & 52336,0 & 58274,0 & 0,22 & 0,22 & 0,23 \\
\hline 3. Sonstige öffentliche Beiträge ${ }^{3}$ & $-4537,0$ & $-549,0$ & $-45,0$ & $-0,02$ & 0,00 & 0,00 \\
\hline $\begin{array}{l}\text { 4. Geschenke privater } \\
\text { Hilfsorganisationen }\end{array}$ & 6934,0 & 7289,0 & 8765,0 & 0,03 & 0,03 & 0,04 \\
\hline Insgesamt & 134259,0 & 108821,0 & 85893,0 & 0,56 & 0,46 & 0,35 \\
\hline \multicolumn{7}{|l|}{ davon: } \\
\hline zu Marktbedingungen $(=1+3)$ & 73591,0 & 49196,0 & 18854,0 & 0,31 & 0,21 & 0,08 \\
\hline zu liberalen Bedingungen $(=2+4)$ & 60668,0 & 59625,0 & 67039,0 & 0,25 & 0,25 & 0,27 \\
\hline \multicolumn{7}{|c|}{ In Milliarden Dollars } \\
\hline BVE der DAC-Mitgliedsländer & 24055,0 & 23815,0 & 24825,0 & & & \\
\hline
\end{tabular}

Quelle: OECD, Statistical Annex of the 2003 Development Co-operation Report, Paris, Januar 2004.

$\mathrm{BVE}=$ Bruttovolkseinommen.

Anmerkungen 1-4: siehe nächste Seite. 
Anmerkung: Die Kategorien der in der Tabelle aufgeführten Finanzflüsse wurden vom Entwicklungshilfeausschuss der OECD festgelegt.

1. Die Privatkapitalströme sind von der Privatwirtschaft zu Marktbedingungen bereitgestellte Finanzmittel.

$\square$ Direktinvestitionen: Investitionen, die zum Zweck getätigt werden, Anteile an einem Unternehmen eines anderen Landes zu erwerben oder dauerhaft zu erhöhen. Die Direktinvestitionsströme entsprechen der Veränderung der Nettoaktiven, welche die Tochtergesellschaften in der Rechnungslegung der Muttergesellschaft darstellen. Sie gehen nicht unbedingt mit Kapitaltransfers im Sinne der vom Internationalen Währungsfonds festgelegten Zahlungsbilanzdefinition einher. Die Statistik beruht auf den Angaben der Unternehmen.

๖ Die Exportkredite sind Darlehen mit einer Laufzeit von über einem Jahr, die für die Anschaffung von Waren im Kreditgeberland gewährt werden. Die in der Tabelle aufgeführten Beträge betreffen nur garantierte Darlehen.

$\square$ Die Wertpapieranlagen umfassen zwei Arten von Finanzflüssen:

a) Bilaterale Anlagen: Zeichnung von Obligationen, die von Drittweltstaaten oder in Entwicklungsländern niedergelassenen Firmen ausgegeben werden, Annahme von Schuldscheinen, Erwerb von Aktien, Obligationen und Immobilien.

b) Multilaterale Anlagen: Anlagen des privaten Banken- und Nichtbankensektors in von multilateralen Institutionen ausgegebene Wertpapiere.

๑ Die Bankflüsse, welche die jährliche Änderung der Nettoguthaben der Inlandsbanken (einschliesslich der Niederlassungen im Ausland) gegenüber den Entwicklungsländern darstellen, sind nicht berücksichtigt.

2. Die öffentliche Entwicklungshilfe stellt die gesamten Finanzflüsse dar, die für jene Länder und Gebiete bereitgestellt werden, welche im 1. Teil der DAC-Liste der Hilfeempfängerländer aufgeführt sind (Entwicklungsländer). Die öffentliche Entwicklungshilfe umfasst auch die Beiträge an die multilateralen Finanzinstitutionen, die aus öffentlichen Mitteln stammen (im Fall der Schweiz einschliesslich der Leistungen der Kantone und Gemeinden). Diese Leistungen werden hauptsächlich mit dem Ziel erbracht, die wirtschaftliche Entwicklung der Entwicklungsländer zu fördern, und werden zu Vorzugsbedingungen gewährt. Bezüglich der Zusammensetzung der öffentlichen Entwicklungshilfe verweisen wir auf die Tabellen im 3. Teil.

Der in der DAC-Statistik aufgeführte Betrag der öffentlichen Entwicklungshilfe der Schweiz kann vom entsprechenden Betrag in der schweizerischen Statistik verschieden sein, und zwar aus folgenden Gründen :

a) Zum einen finanziert die Schweiz gewisse internationale Beiträge durch die Emission von Schuldscheinen (Notes), deren Einlösung zu einem späteren Zeitpunkt erfolgt. Diese Verpflichtungen sind im Betrag der Hilfe enthalten, welcher der OECD zur Zeit der Verpflichtung gemeldet wird, wogegen die Schweiz einen Hilfebeitrag auf der Grundlage der im Jahr effektiv geleisteten Auszahlungen veröffentlicht.

b) Zum anderen können sich auch Unterschiede beim Betrag der öffentlichen Entwicklungshilfe der Schweiz aus statistischen Überarbeitungen im Laufe des Jahres ergeben. Die Quellen einiger Tabellen können aus dem Jahr 2003 sein (Jahresbericht der internationalen Zusammenarbeit, DEZA/seco) oder sich aus Zahlen ergeben, die von der DEZA zu einem späteren Zeitpunkt (Februar 2004) übermittelt wurden. Dies erklärt die Tatsache, dass einige Zahlen revidiert wurden.

3. Die sonstigen Beiträge des öffentlichen Sektors umfassen alle anderen Mittel, die den Entwicklungsländern von den öffentlichen Körperschaften zur Verfügung gestellt, jedoch nicht zu Vorzugsbedingungen gewährt werden.

4. Die Geschenke privater Hilfsorganisationen umfassen die von Institutionen ohne Erwerbszweck erbrachten Leistungen (ohne die vom öffentlichen Sektor erhaltenen Subventionen).

Das Bruttovolkseinkommen (BVE) der Schweiz ist zu (laufenden) Marktpreisen geschätzt. 


\subsection{Beziehungen zwischen Bruttovolkseinkommen (BVE), Privatkapitalnettoflüssen und öffentlicher Entwicklungshilfe}

\section{Vergleich zwischen den Mitgliedsländern des DAC (2002)}

\begin{tabular}{|c|c|c|c|c|c|c|c|c|c|c|}
\hline \multirow{3}{*}{$\begin{array}{l}\text { Mitgliedsländer } \\
\text { des Entwicklungs- } \\
\text { hilfeausschusses } \\
\text { der OECD }\end{array}$} & \multicolumn{3}{|c|}{$\begin{array}{c}\text { Bruttovolkseinkommen } \\
\text { (BVE) }\end{array}$} & \multicolumn{3}{|c|}{$\begin{array}{l}\text { Privatkapital- } \\
\text { nettoflüsse }^{1}\end{array}$} & \multicolumn{4}{|c|}{$\begin{array}{l}\text { Nettoflüsse der öffentlichen } \\
\text { Entwicklungshilfe }^{2}\end{array}$} \\
\hline & \multirow{2}{*}{$\frac{\text { Betrag }}{\text { Mia } \$}$} & \multicolumn{2}{|c|}{ pro Einwohner } & \multirow{2}{*}{$\begin{array}{r}\text { Betrag } \\
\text { Mio \$ }\end{array}$} & \multicolumn{2}{|c|}{ In \% des BVE } & \multirow{2}{*}{$\begin{array}{c}\text { Betrag } \\
\text { Mio\$ }\end{array}$} & \multicolumn{3}{|c|}{ In \% des BVE } \\
\hline & & $\%$ & $\$$ & & $\%$ & Rang & & $\%$ & $\%$ & Rang \\
\hline USA & 10490 & 42,3 & 36400 & 5173 & 0,05 & 8 & 13290 & 22,81 & 0,13 & 22 \\
\hline Japan & 4065 & 16,4 & 31900 & -573 & $-0,01$ & 13 & 9283 & 15,93 & 0,23 & 18 \\
\hline Deutschland & 1987 & 8,0 & 24100 & -1124 & $-0,06$ & 16 & 5324 & 9,14 & 0,27 & 14 \\
\hline Grossbritannien & 1595 & 6,4 & 27000 & 13547 & 0,85 & 3 & 4924 & 8,45 & 0,31 & 11 \\
\hline Frankreich & 1439 & 5,8 & 24200 & -1392 & $-0,10$ & 17 & 5486 & 9,41 & 0,38 & 8 \\
\hline Italien & 1174 & 4,7 & 20300 & -563 & $-0,05$ & 15 & 2332 & 4,00 & 0,20 & 21 \\
\hline Kanada & 718 & 2,9 & 22800 & 188 & 0,03 & 12 & 2006 & 3,44 & 0,28 & 12 \\
\hline Spanien & 652 & 2,6 & 15800 & 6404 & 0,98 & 2 & 1712 & 2,94 & 0,26 & 15 \\
\hline Niederlande & 412 & 1,7 & 25500 & -5310 & $-1,29$ & 21 & 3338 & 5,73 & 0,81 & 4 \\
\hline Australien & 387 & 1,6 & 19800 & -433 & $-0,11$ & 18 & 989 & 1,70 & 0,26 & 16 \\
\hline Schweiz & 297 & 1,2 & 40600 & 1089 & 0,37 & 5 & 939 & 1,61 & 0,32 & 10 \\
\hline Belgien & 248 & 1,0 & 24000 & 86 & 0,03 & 9 & 1072 & 1,84 & 0,43 & 6 \\
\hline Schweden & 239 & 1,0 & 26700 & 199 & 0,08 & 6 & 1991 & 3,42 & 0,83 & 3 \\
\hline Österreich & 204 & 0,8 & 25400 & 1325 & 0,65 & 4 & 520 & 0,89 & 0,26 & 17 \\
\hline Norwegen & 191 & 0,8 & 42000 & 131 & 0,07 & 7 & 1696 & 2,91 & 0,89 & 2 \\
\hline Dänemark & 170 & 0,7 & 31700 & -63 & $-0,04$ & 14 & 1643 & 2,82 & 0,96 & 1 \\
\hline Griechenland & 133 & 0,5 & 12200 & 40 & 0,03 & 10 & 276 & 0,47 & 0,21 & 20 \\
\hline Finnland & 131 & 0,5 & 25200 & -676 & $-0,52$ & 20 & 462 & 0,79 & 0,35 & 9 \\
\hline Portugal & 119 & 0,5 & 11500 & -150 & $-0,13$ & 19 & 323 & 0,55 & 0,27 & 13 \\
\hline Irland & 99 & 0,4 & 25500 & 986 & 1,00 & 1 & 398 & 0,68 & 0,40 & 7 \\
\hline Neuseeland & 55 & 0,2 & 13900 & 17 & 0,03 & 11 & 122 & 0,21 & 0,22 & 19 \\
\hline Luxemburg & 19 & 0,1 & 43300 & - & - & - & 147 & 0,25 & 0,77 & 5 \\
\hline $\begin{array}{l}\text { Gesamtbetrag } \\
\text { oder Durchschnitt }\end{array}$ & 24825 & 100,0 & 28800 & 18899 & 0,08 & & 58274 & 100,00 & 0,23 & \\
\hline
\end{tabular}

Quelle: OECD, Statistical Annex of the 2003 Development Co-operation Report, Paris, Januar 2004.

Anmerkung: Siehe auch die Grafiken 9 und 10 im 3. Teil „Öffentliche Entwicklungshilfe“.

1 Einschliesslich der zur Streichung von Handelsforderungen bestimmten Beträge. Der Erlass von Militärforderungen wird nicht berücksichtigt.

2 Die öffentliche Entwicklungshilfe Frankreichs umfasst die Hilfe für die Übersee-Gebiete. Hingegen schliesst die Statistik die Transfers an die Übersee-Departemente aus. 


\section{3. Übersicht über die Finanzbeziehungen der Schweiz zur restlichen Welt}

\section{A. Anleihen ausländischer Schuldner nach Ländergruppen, in Millionen Franken ${ }^{1}$}

1. 2002

\begin{tabular}{lrrr}
\hline Ländergruppen & $\begin{array}{c}\text { Emissions- } \\
\text { wert }\end{array}$ & Rückzahlung & $\begin{array}{r}\text { Nettoentnahme } \\
\text { auf dem } \text { Markt }^{2}\end{array}$ \\
\hline Europäische Union & 29068,9 & 15310,6 & 13758,3 \\
\hline Übriges Europa & 4003,0 & 2061,7 & 1941,3 \\
\hline USA, Kanada & 5292,5 & 6789,0 & $-1496,5$ \\
\hline Karibik & 3636,0 & 2742,3 & 893,7 \\
\hline Lateinamerika & 0,0 & 0,0 & 0,0 \\
\hline Mittlerer Osten, Afrika & 0,0 & 0,0 & 0,0 \\
\hline Japan & 0,0 & 1100,0 & $-1100,0$ \\
\hline Australien, Neuseeland & 1048,0 & 200,0 & 048,0 \\
\hline Übrige Länder Asiens, Ozeanien & 0,0 & & $-31,7$ \\
\hline $\begin{array}{l}\text { Internationale Entwicklungs- } \\
\text { organisationen }\end{array}$ & 693,3 & 725,0 & $\mathbf{1 4 8 1 3 , 1}$ \\
\hline Gesamtbetrag & $\mathbf{4 3 7 4 1 , 7}$ & $\mathbf{2 8 9 2 8 , 6}$ &
\end{tabular}

\section{2003}

\begin{tabular}{lrrr}
\hline Ländergruppen & $\begin{array}{c}\text { Emissions- } \\
\text { wert }\end{array}$ & Rückzahlung & $\begin{array}{c}\text { Nettoentnahme } \\
\text { auf dem } \text { Markt }^{2}\end{array}$ \\
\hline Europäische Union & 34642,6 & 17852,3 & 16790,3 \\
\hline Übriges Europa & 5723,6 & 2197,8 & 3525,8 \\
\hline USA, Kanada & 3366,7 & 6248,0 & $-2881,3$ \\
\hline Karibik & 3850,5 & 5633,5 & $-1783,0$ \\
\hline Lateinamerika & 0,0 & 0,0 & 0,0 \\
\hline Mittlerer Osten, Afrika & 0,0 & 0,0 & 0,0 \\
\hline Japan & 0,0 & 850,0 & $-850,0$ \\
\hline Australien, Neuseeland & 601,7 & 500,0 & 101,7 \\
\hline Übrige Länder Asiens, Ozeanien & 0,0 & 0,0 & 0,0 \\
\hline Internationale Entwicklungs- & 301,8 & 1100,0 & $-798,2$ \\
\hline organisationen & $\mathbf{3}$ & $\mathbf{3 4 3 8 1 , 6}$ & $\mathbf{1 4 1 0 5 , 3}$ \\
\hline Gesamtbetrag & $\mathbf{4 8 4 8 6 , 9}$ & &
\end{tabular}

Quelle: Schweizerische Nationalbank, Statistisches Monatsheft, Januar 2004.

Öffentliche Emissionen.

2 Emissionswert abzüglich Rückzahlungen.

3 Weltbankgruppe (IBRD, IDA, SFI, MIGA), Interamerikanische Entwicklungsbank (IDB), Afrikanische Entwicklungsbank (BAD), Asiatische Entwicklungsbank (ADB), Europäische Bank für Wiederaufbau und Entwicklung (EBWE). 


\section{3. Übersicht über die Finanzbeziehungen der Schweiz zur restlichen Welt (Fortsetzung)}
B. Auslandsguthaben und -verpflichtungen der in der Schweiz niedergelassenen Banken

\section{In den Bilanzen aufgeführte Guthaben und Verpflichtungen Stand am 31. Dezember 2002}

\begin{tabular}{|c|c|c|c|c|}
\hline \multirow[t]{2}{*}{ Land $^{3}$} & $\begin{array}{c}\text { Guthaben } \\
\text { A }\end{array}$ & $\begin{array}{l}\text { Verpflichtungen } \\
\text { B }\end{array}$ & $\begin{array}{l}\text { Saldo } \\
\text { C }=A-B\end{array}$ & $\begin{array}{r}\text { Deckung } \\
\mathrm{D}=\mathrm{A} / \mathrm{B}\end{array}$ \\
\hline & \multicolumn{3}{|c|}{ In Millionen Franken } & Guth./Verpfl. \\
\hline Industrieländer $^{3}$ & 1179831 & 962558 & 217273 & 1,23 \\
\hline MOEL/NUS & 4956 & 4132 & 824 & 1,20 \\
\hline \multicolumn{2}{|c|}{$\begin{array}{l}\text { Entwicklungsländer und } \\
\text { fortgeschr. Entwicklungsländer (EL) } \\
131631\end{array}$} & 226772 & -95141 & 0,58 \\
\hline EL Europa & 6335 & 8762 & -2427 & 0,72 \\
\hline EL Amerika & 63498 & 102579 & -39081 & 0,62 \\
\hline EL Afrika & 5585 & 12101 & -6516 & 0,46 \\
\hline EL Asien, Pazifik & 56213 & 103330 & -47117 & 0,54 \\
\hline \multicolumn{5}{|l|}{ Aufteilung nach Ländern: } \\
\hline Europäische Union und EFTA & 662082 & 523371 & 138711 & 1,27 \\
\hline Grossbritannien & 328151 & 247197 & 80954 & 1,33 \\
\hline Deutschland & 98482 & 65210 & 33272 & 1,51 \\
\hline Frankreich & 72020 & 63350 & 8670 & 1,14 \\
\hline Belgien & 20440 & 32068 & -11628 & 0,64 \\
\hline Italien & 39171 & 31614 & 7557 & 1,24 \\
\hline Luxemburg & 19302 & 30114 & -10812 & 0,64 \\
\hline Niederlande & 23858 & 13836 & 10022 & 1,72 \\
\hline Spanien & 10806 & 10591 & 215 & 1,02 \\
\hline Übrige Länder & 49852 & 29391 & 20461 & 1,70 \\
\hline MOEL/NUS & 4956 & 4132 & 824 & 1,20 \\
\hline Russland & 2845 & 2307 & 538 & 1,23 \\
\hline Polen & 656 & 351 & 305 & 1,87 \\
\hline Tschechische Republik & 237 & 343 & -106 & 0,69 \\
\hline Ukraine & 34 & 332 & -298 & 0,10 \\
\hline Übrige Länder & 1184 & 799 & 385 & 1,48 \\
\hline EL Europa & 6335 & 8762 & -2427 & 0,72 \\
\hline Türkei & 2356 & 3279 & -923 & 0,72 \\
\hline Gibraltar & 1004 & 2264 & -1260 & 0,44 \\
\hline Zypern & 1888 & 1652 & 236 & 1,14 \\
\hline Serbien und Montenegro & 51 & 562 & -511 & 0,09 \\
\hline Kroatien & 86 & 521 & -435 & 0,17 \\
\hline Übrige Länder & 950 & 484 & 466 & 1,96 \\
\hline Übriges Europa & 47333 & 137101 & -89768 & 0,35 \\
\hline Jersey & 41904 & 65194 & -23290 & 0,64 \\
\hline Guernsey & 2339 & 60502 & -58163 & 0,04 \\
\hline Monaco & 2319 & 9664 & -7345 & 0,24 \\
\hline Insel Man & 704 & 1425 & -721 & 0,49 \\
\hline Übrige Länder & 67 & 316 & -249 & 0,21 \\
\hline
\end{tabular}




\begin{tabular}{|c|c|c|c|c|}
\hline \multirow[t]{2}{*}{ Land $^{3}$} & $\begin{array}{c}\text { Guthaben } \\
\text { A }\end{array}$ & $\begin{array}{l}\text { Verpflichtungen } \\
\text { B }\end{array}$ & $\begin{array}{c}\text { Saldo } \\
\text { C }=A-B\end{array}$ & \multirow{2}{*}{$\begin{array}{r}\begin{array}{r}\text { Deckung } \\
\mathrm{D}=\mathrm{A} / \mathrm{B}\end{array} \\
\text { Guth./Verpfl. }\end{array}$} \\
\hline & \multicolumn{3}{|c|}{ In Millionen Franken } & \\
\hline USA, Kanada & 409292 & 259296 & 149996 & 1,58 \\
\hline EL Nord- und Mittelamerika & 57225 & 90187 & -32962 & 0,63 \\
\hline Brit. Antillen & 13073 & 27824 & -14751 & 0,47 \\
\hline Cayman-Inseln & 25072 & 26622 & -1550 & 0,94 \\
\hline Bahamas & 3781 & 11668 & -7887 & 0,32 \\
\hline Panama & 3206 & 9524 & -6318 & 0,34 \\
\hline Bermudas & 4776 & 7113 & -2337 & 0,67 \\
\hline Niederl. Antillen & 1237 & 1909 & -672 & 0,65 \\
\hline Mexiko & 3871 & 1720 & 2151 & 2,25 \\
\hline Antigua und Barbuda & 252 & 968 & -716 & 0,26 \\
\hline Belize & 193 & 685 & -492 & 0,28 \\
\hline Turks- und Caicos-Inseln & 82 & 365 & -283 & 0,22 \\
\hline Übrige Länder & 1682 & 1789 & -107 & 0,94 \\
\hline Südamerika & 6273 & 12392 & -6119 & 0,51 \\
\hline Brasilien & 2438 & 4807 & -2369 & 0,51 \\
\hline Argentinien & 875 & 2635 & -1760 & 0,33 \\
\hline Venezuela & 1067 & 2444 & -1377 & 0,44 \\
\hline Urugay & 187 & 744 & -557 & 0,25 \\
\hline Peru & 346 & 529 & -183 & 0,65 \\
\hline Chile & 266 & 496 & -230 & 0,54 \\
\hline Kolumbien & 844 & 368 & 476 & 2,29 \\
\hline Übrige Länder & 250 & 369 & -119 & 0,68 \\
\hline Afrika & 5585 & 12101 & -6516 & 0,46 \\
\hline Liberia & 1712 & 2116 & -404 & 0,81 \\
\hline Südafrika & 1208 & 2084 & -876 & 0,58 \\
\hline Ägypten & 710 & 1882 & -1172 & 0,38 \\
\hline Libyen & 127 & 1334 & -1207 & 0,10 \\
\hline Kenia & 229 & 769 & -540 & 0,30 \\
\hline Marokko & 244 & 593 & -349 & 0,41 \\
\hline Elfenbeinküste & 316 & 394 & -78 & 0,80 \\
\hline Nigeria & 97 & 357 & -260 & 0,27 \\
\hline Sudan & 90 & 336 & -246 & 0,27 \\
\hline Tunesien & 51 & 325 & -274 & 0,16 \\
\hline Übrige Länder & 801 & 1911 & -1110 & 0,42 \\
\hline Mittlerer Osten & 12826 & 41794 & -28968 & 0,31 \\
\hline Syrien & 68 & 14764 & -14696 & 0,00 \\
\hline Saudi-Arabien & 3491 & 6608 & -3117 & 0,53 \\
\hline Verein. Arab. Emirate & 1672 & 5428 & -3756 & 0,31 \\
\hline Israel & 1750 & 4449 & -2699 & 0,39 \\
\hline Jordanien & 208 & 2199 & -1991 & 0,09 \\
\hline Kuweit & 1218 & 2142 & -924 & 0,57 \\
\hline Libanon & 794 & 1950 & -1156 & 0,41 \\
\hline Iran & 1222 & 1914 & -692 & 0,64 \\
\hline Bahrain & 1800 & 871 & 929 & 2,07 \\
\hline Jemen & 232 & 644 & -412 & 0,36 \\
\hline Irak & 169 & 386 & -217 & 0,44 \\
\hline Übrige Länder & 202 & 439 & -237 & 0,46 \\
\hline
\end{tabular}




\begin{tabular}{|c|c|c|c|c|}
\hline \multirow[t]{2}{*}{ Land $^{3}$} & $\begin{array}{c}\text { Guthaben } \\
\text { A }\end{array}$ & $\begin{array}{c}\text { Verpflichtungen } \\
\text { B }\end{array}$ & $\begin{array}{c}\text { Saldo } \\
\text { C = A-B }\end{array}$ & \multirow{2}{*}{$\begin{array}{r}\begin{array}{r}\text { Deckung } \\
\mathrm{D}=\mathrm{A} / \mathrm{B}\end{array} \\
\text { Guth./Verpfl. }\end{array}$} \\
\hline & \multicolumn{3}{|c|}{ In Millionen Franken } & \\
\hline Australien, Neuseeland & 8125 & 8587 & -462 & 0,95 \\
\hline Japan & 52999 & 34203 & 18796 & 1,55 \\
\hline EL Asien, Ozeanien & 43387 & 61536 & -18149 & 0,71 \\
\hline Hongkong & 11023 & 20596 & -9573 & 0,54 \\
\hline Singapur & 13370 & 13813 & -443 & 0,97 \\
\hline Taiwan & 1981 & 6080 & -4099 & 0,33 \\
\hline Indonesien & 3062 & 4991 & -1929 & 0,61 \\
\hline Südkorea & 7012 & 4396 & 2616 & 1,60 \\
\hline China & 1239 & 2200 & -961 & 0,56 \\
\hline Pakistan & 769 & 1929 & -1160 & 0,40 \\
\hline Indien & 753 & 1847 & -1094 & 0,41 \\
\hline Malaysia & 1065 & 1472 & -407 & 0,72 \\
\hline Philippinen & 1454 & 1380 & 74 & 1,05 \\
\hline Thailand & 1050 & 1081 & -31 & 0,97 \\
\hline Übrige Länder & 609 & 1751 & -1142 & 0,35 \\
\hline Insgesamt $^{4}$ & 1316420 & 1193463 & 122957 & 1,10 \\
\hline
\end{tabular}

Quelle: Schweizerische Nationalbank, Das schweizerische Bankwesen 2002, 2003.

Anmerkung: Die Schweizerische Nationalbank gibt eine Statistik der Auslandsguthaben und -verpflichtungen der in der Schweiz niedergelassenen Banken heraus. Sie unterscheidet die von den Banken auf eigene Rechnung getätigten (in der Bilanz aufgeführten) Geschäfte (vgl. Tabelle 2.3.B.1) und die auf Rechnung und Risiko der Kunden getätigten Transaktionen (Treuhandgeschäfte, vgl. Tabelle 2.3.B.2). Die Statistik zeigt, dass die Guthaben der Schweizer Banken in den Entwicklungsländern geringer sind als ihre Verpflichtungen gegenüber diesen Ländern. Im Jahr 2002 erreichte die Deckungsrate 58 Prozent.

Fussnoten zu den Tabellen 2.3.B.1 und 2.3.B.2

1 Gemäss Kontenbericht von 110 im Auslandsgeschäft tätigen Banken. Die Guthaben und Verpflichtungen der Niederlassungen (nicht aber der Tochtergesellschaften) im Ausland werden in der Statistik berücksichtigt.

2 Nur ein Teil der Auslandsgeschäfte ist in den Bankbilanzen wiedergegeben. Es handelt sich lediglich um die von den Banken auf eigene Rechnung getätigten Geschäfte. Die für die Kunden erbrachten Dienstleistungsgeschäfte der Banken, wie Vermögensverwaltung, Börsentätigkeit, Emissionen, Treuhandgeschäfte, Devisenhandel, Eröffnung von Akkreditiven, Kautionen usw., erscheinen nicht in der Bilanz.

3 Länderklassifikation gemäss DAC-Liste am Schluss des Jahrbuchs.

4 Ohne Edelmetalle. 


\section{3. Übersicht über die Finanzbeziehungen der Schweiz zur restlichen Welt (Fortsetzung)}

B. Auslandsguthaben und -verpflichtungen der in der Schweiz niedergelassenen Banken'

\section{Treuhandguthaben und -verpflichtungen ${ }^{2}$} Stand am 31. Dezember 2002

\begin{tabular}{|c|c|c|c|c|}
\hline \multirow[t]{2}{*}{ Land $^{3}$} & $\begin{array}{c}\text { Guthaben } \\
\text { A }\end{array}$ & $\begin{array}{l}\text { Verpflichtungen } \\
\text { B }\end{array}$ & $\begin{array}{l}\text { Saldo } \\
\mathrm{C}=\mathrm{A}-\mathrm{B}\end{array}$ & \multirow{2}{*}{$\begin{array}{r}\text { Deckung } \\
\mathrm{D}=\mathrm{A} / \mathrm{B} \\
\text { Guth./Verpfl. }\end{array}$} \\
\hline & \multicolumn{3}{|c|}{ In Millionen Franken } & \\
\hline Industrieländer & 301710 & 107628 & 194082 & 2,80 \\
\hline MOEL/NUS & 377 & 5129 & -4752 & 0,07 \\
\hline $\begin{array}{l}\text { Entwicklungsländer und } \\
\text { fortgeschr. Entwicklungsländer (EL }\end{array}$ & L) 12596 & 154484 & -141888 & 0,08 \\
\hline EL Europa & 781 & 7929 & -7148 & 0,10 \\
\hline EL Amerikas & 9359 & 88957 & -79598 & 0,11 \\
\hline EL Afrika & 279 & 11288 & -11009 & 0,02 \\
\hline EL Asien, Pazifiken & 2177 & 46310 & -44133 & 0,05 \\
\hline \multicolumn{5}{|l|}{ Aufteilung nach Ländern: } \\
\hline Europäische Union und EFTA & 239162 & 73201 & 165961 & 3,27 \\
\hline Grossbritannien & 43014 & 15398 & 27616 & 2,79 \\
\hline Italien & 649 & 13048 & -12399 & 0,05 \\
\hline Frankreich & 35075 & 9930 & 25145 & 3,53 \\
\hline Deutschland & 11480 & 8787 & 2693 & 1,31 \\
\hline Spanien & 1427 & 7340 & -5913 & 0,19 \\
\hline Belgien & 23806 & 5632 & 18174 & 4,23 \\
\hline Griechenland & 0 & 4466 & -4466 & 0,00 \\
\hline Niederlande & 41795 & 2204 & 39591 & 18,96 \\
\hline Luxemburg & 76747 & 2165 & 74582 & 35,45 \\
\hline Portugal & 357 & 1445 & -1088 & 0,25 \\
\hline Übrige Länder & 4812 & 2786 & 2026 & 1,73 \\
\hline MOEL/NUS & 377 & 5129 & -4752 & 0,07 \\
\hline Russland & 168 & 3858 & -3690 & 0,04 \\
\hline Ukraine & 0 & 267 & -267 & 0,00 \\
\hline Polen & 10 & 237 & -227 & 0,04 \\
\hline Übrige Länder & 199 & 767 & -568 & 0,26 \\
\hline EL Europa & 781 & 7929 & -7148 & 0,10 \\
\hline Türkei & 480 & 4442 & -3962 & 0,11 \\
\hline Gibraltar & 0 & 1887 & -1887 & 0,00 \\
\hline Zypern & 61 & 914 & -853 & 0,07 \\
\hline Malta & 240 & 338 & -98 & 0,71 \\
\hline Übrige Länder & 0 & 348 & -348 & 0,00 \\
\hline Übriges Europa & 58443 & 24360 & 34083 & 2,40 \\
\hline Jersey & 22076 & 13878 & 8198 & 1,59 \\
\hline Insel Man & 0 & 5390 & -5390 & 0,00 \\
\hline Monaco & 591 & 2681 & -2090 & 0,22 \\
\hline Guernsey & 31310 & 2205 & 29105 & 14,20 \\
\hline Übrige Länder & 4466 & 206 & 4260 & 21,68 \\
\hline
\end{tabular}




\begin{tabular}{lcccr}
\hline & Guthaben & Verpflichtungen & Saldo & Deckung \\
Land $^{3}$ & A & B & C =A-B & D = A/B \\
\hline
\end{tabular}

In Millionen Franken

Guth./Verpfl.

\begin{tabular}{|c|c|c|c|c|}
\hline USA, Kanada & 3437 & 7823 & -4386 & 0,44 \\
\hline EL Nord- und Mittelamerika. & 9127 & 73503 & -64376 & 0,12 \\
\hline Brit. Antillen & 374 & 31742 & -31368 & 0,01 \\
\hline Panama & 84 & 16008 & -15924 & 0,01 \\
\hline Cayman-Inseln & 3833 & 7262 & -3429 & 0,53 \\
\hline Bahamas & 4010 & 6242 & -2232 & 0,64 \\
\hline Mexiko & 26 & 3270 & -3244 & 0,01 \\
\hline Niederl. Antillen & 469 & 2988 & -2519 & 0,16 \\
\hline Bermudas & 0 & 1828 & -1828 & 0,00 \\
\hline Belize & 0 & 908 & -908 & 0,00 \\
\hline Turks- und Caicos-Inseln & 0 & 766 & -766 & 0,00 \\
\hline St-Vincent & 0 & 526 & -526 & 0,00 \\
\hline Übrige Länder & 331 & 1963 & -1632 & 0,17 \\
\hline Südamerika & 232 & 15454 & -15222 & 0,02 \\
\hline Brasilien & 112 & 5137 & -5025 & 0,02 \\
\hline Argentinien & 37 & 4765 & -4728 & 0,01 \\
\hline Venezuela & 0 & 2668 & -2668 & 0,00 \\
\hline Uruguay & 0 & 1227 & -1227 & 0,00 \\
\hline Kolumbien & 0 & 435 & -435 & 0,00 \\
\hline Chile & 30 & 402 & -372 & 0,07 \\
\hline Ecuador & 0 & 319 & -319 & 0,00 \\
\hline Peru & 22 & 242 & -220 & 0,09 \\
\hline Paraguay & 0 & 221 & -221 & 0,00 \\
\hline Übrige Länder & 31 & 38 & -7 & 0,82 \\
\hline Afrika & 279 & 11288 & -11009 & 0,02 \\
\hline Liberia & 0 & 2372 & -2372 & 0,00 \\
\hline Ägypten & 0 & 2305 & -2305 & 0,00 \\
\hline Marokko & 0 & 985 & -985 & 0,00 \\
\hline Nigeria & 0 & 780 & -780 & 0,00 \\
\hline Südafrika & 22 & 779 & -757 & 0,03 \\
\hline Kenia & 0 & 710 & -710 & 0,00 \\
\hline Angola & 0 & 611 & -611 & 0,00 \\
\hline Libyen & 0 & 402 & -402 & 0,00 \\
\hline Mauritius & 0 & 389 & -389 & 0,00 \\
\hline Algerien & 0 & 272 & -272 & 0,00 \\
\hline Tunesien & 0 & 252 & -252 & 0,00 \\
\hline Elfenbeinküste & 0 & 230 & -230 & 0,00 \\
\hline Übrige Länder & 257 & 1201 & -944 & 0,21 \\
\hline
\end{tabular}




\begin{tabular}{|c|c|c|c|c|}
\hline \multirow[t]{2}{*}{ Land } & $\begin{array}{c}\text { Guthaben } \\
\text { A }\end{array}$ & $\begin{array}{l}\text { Verpflichtungen } \\
\text { B }\end{array}$ & $\begin{array}{c}\text { Saldo } \\
\mathrm{C}=\mathrm{A}-\mathrm{B}\end{array}$ & \multirow{2}{*}{$\begin{array}{r}\text { Deckung } \\
\mathrm{D}=\mathrm{A} / \mathrm{B}\end{array}$} \\
\hline & \multicolumn{3}{|c|}{ In Millionen Franken } & \\
\hline Mittlerer-Osten & 629 & 32742 & -32113 & 0,02 \\
\hline Saudi-Arabien & 0 & 10300 & -10300 & 0,00 \\
\hline Verein. Arab. Emirate & 0 & 8075 & -8075 & 0,00 \\
\hline Libanon & 376 & 4151 & -3775 & 0,09 \\
\hline Israel & 0 & 3844 & -3844 & 0,00 \\
\hline Jordanien & 0 & 1734 & -1734 & 0,00 \\
\hline Kuweit & 0 & 1263 & -1263 & 0,00 \\
\hline Bahrain & 0 & 882 & -882 & 0,00 \\
\hline Iran & 0 & 696 & -696 & 0,00 \\
\hline Syrien & 0 & 689 & -689 & 0,00 \\
\hline Jemen & 0 & 484 & -484 & 0,00 \\
\hline Oman & 0 & 284 & -284 & 0,00 \\
\hline Übrige Länder & 253 & 340 & -87 & 0,74 \\
\hline Australien, Neuseeland & 24 & 1356 & -1332 & 0,02 \\
\hline Japan & 644 & 888 & -244 & 0,73 \\
\hline EL Asien, Ozeanien & 1548 & 13568 & -12020 & 0,11 \\
\hline Hongkong & 462 & 4585 & -4123 & 0,10 \\
\hline Taiwan & 0 & 1427 & -1427 & 0,00 \\
\hline Singapur & 1043 & 1348 & -305 & 0,77 \\
\hline Indien & 0 & 1086 & -1086 & 0,00 \\
\hline Pakistan & 0 & 1036 & -1036 & 0,00 \\
\hline Thailand & 0 & 716 & -716 & 0,00 \\
\hline Indonesien & 0 & 635 & -635 & 0,00 \\
\hline Philippinen & 0 & 554 & -554 & 0,00 \\
\hline Malaysia & 0 & 435 & -435 & 0,00 \\
\hline Südkorea & 0 & 244 & -244 & 0,00 \\
\hline Marshall-Inseln & 0 & 206 & -206 & 0,00 \\
\hline Übrige Länder & 43 & 1296 & -1253 & 0,03 \\
\hline Insgesamt $^{4}$ & 314684 & 267247 & 47437 & 1,18 \\
\hline
\end{tabular}

Quelle: Schweizerische Nationalbank, Das schweizerische Bankwesen 2002, 2003.

Siehe Fussnoten am Schluss von Tabelle 2.3.B.1. 


\subsection{Schweizerische Direktinvestitionen im Ausland, in Millionen Franken}

\section{A. Geografische Aufteilung der Investitionsflüsse und des Kapitalbestands nach Jahren}

\begin{tabular}{|c|c|c|c|c|c|c|}
\hline \multirow{2}{*}{ Land } & \multicolumn{3}{|c|}{ Investitionsflüsse } & \multicolumn{3}{|c|}{ Kapitalbestand } \\
\hline & 2001 & 2002 & $\begin{array}{c}\text { Absol. Veränd. } \\
02 / 01\end{array}$ & 2001 & 2002 & $\begin{array}{l}\text { Veränd. } \\
02 / 01 \text { in \% }\end{array}$ \\
\hline $\begin{array}{l}\text { Industrieländer, MOEL/NUS } \\
\text { und andere europ. Länder }{ }^{1}\end{array}$ & 23735 & 12611 & -11124 & 327049 & 309335 & $-\mathbf{5 , 7}$ \\
\hline $\begin{array}{l}\text { Nichteurop. Entwicklungsländer } \\
\text { und fortg. Entwicklungsländer }\end{array}$ & 7048 & -800 & -7848 & 98232 & 100320 & 2,1 \\
\hline Asien $^{2}$ & 700 & 927 & -177 & 28926 & 29777 & 2,9 \\
\hline Lateinamerika & 6205 & -1236 & -7441 & 64903 & 67047 & 3,3 \\
\hline Afrika & 143 & -491 & -634 & 4403 & 3496 & $-20,6$ \\
\hline \multicolumn{7}{|l|}{ Aufteilung nach Ländern: } \\
\hline Europäische Union & 11632 & 7271 & -4361 & 180530 & 179839 & $-0,4$ \\
\hline Belgien & -545 & 2030 & 2575 & 10823 & 11949 & 10,4 \\
\hline Dänemark & -29 & 56 & 85 & 1226 & 1298 & 5,9 \\
\hline Deutschland & 5696 & 240 & -5456 & 27630 & 27673 & 0,2 \\
\hline Finnland & 99 & -7 & -106 & 2197 & 2015 & $-8,3$ \\
\hline Frankreich & -1582 & 1076 & 2658 & 17495 & 20110 & 14,9 \\
\hline Griechenland & 167 & 241 & 74 & 1821 & 1748 & $-4,0$ \\
\hline Irland & -4713 & 147 & 4860 & 10255 & 7836 & $-23,6$ \\
\hline Italien & 1958 & 773 & -1185 & 8828 & 10039 & 13,7 \\
\hline Luxemburg & 7825 & -2956 & -10781 & 24501 & 18746 & $-23,5$ \\
\hline Niederlande & 167 & 1505 & 1338 & 17845 & 19951 & 11,8 \\
\hline Österreich & 399 & 431 & 32 & 4782 & 4636 & $-3,1$ \\
\hline Portugal & 270 & -28 & -298 & 1821 & 1849 & 1,5 \\
\hline Schweden & -165 & 2995 & 3160 & 5090 & 4855 & $-4,6$ \\
\hline Spanien & 1382 & 480 & -902 & 6408 & 7771 & 21,3 \\
\hline Grossbritannien $^{3}$ & 703 & 288 & -415 & 39808 & 39363 & $-1,1$ \\
\hline Übriges Europa & 1775 & 2564 & 789 & 3437 & 33232 & $-3,3$ \\
\hline EFTA & 247 & 791 & 544 & 3679 & 3180 & $-13,6$ \\
\hline Kroatien & 21 & 7 & -14 & 241 & 157 & $-34,9$ \\
\hline Polen & 209 & -152 & -361 & 2469 & 2296 & $-7,0$ \\
\hline Russische Föderation & 696 & 151 & -545 & 1597 & 1362 & $-14,7$ \\
\hline Tschechische Republik & 274 & 55 & -219 & 1952 & 2011 & 3,0 \\
\hline Slowakei & 9 & 53 & 44 & 166 & 180 & 8,4 \\
\hline Ungarn & 170 & 28 & -142 & 1228 & 660 & $-46,3$ \\
\hline Türkei & -158 & 246 & 404 & 1010 & 1042 & 3,2 \\
\hline USA, Kanada & 9728 & 3238 & -6490 & 104742 & 83982 & $-19,8$ \\
\hline Andere nichteurop. entw. Länder & r 442 & -216 & -658 & 8416 & 13324 & 58,3 \\
\hline Australien, Neuseeland & -44 & 424 & 221 & 3743 & 5407 & 44,5 \\
\hline Japan & 487 & -640 & -1127 & 4673 & 7917 & 69,4 \\
\hline
\end{tabular}

${ }^{1}$ ohne Türkei.

2 mit Türkei.

${ }^{3}$ einschliesslich Guernsey, Jersey und Insel Man. 
Investitionsflüsse

Land

\begin{tabular}{|c|c|c|c|c|c|c|}
\hline & 2001 & 2002 & $\begin{array}{c}\text { Absol. Veränd. } \\
02 / 01\end{array}$ & 2001 & 2002 & $\begin{array}{l}\text { Veränd. } \\
02 / 01 \text { in } \%\end{array}$ \\
\hline Asien & 858 & 681 & -177 & 27916 & 28735 & 2,9 \\
\hline Hongkong & 258 & -202 & -460 & 2825 & 2936 & 3,9 \\
\hline Südkorea & 86 & -11 & -9 & 831 & 1122 & 35,0 \\
\hline Malaysia & 67 & -34 & -101 & 1399 & 1163 & $-16,9$ \\
\hline Philippinen & 98 & -272 & -370 & 1999 & 2044 & 2,3 \\
\hline Singapur & -523 & 1037 & 1560 & 13056 & 14345 & 9,9 \\
\hline Taiwan & 34 & 31 & -3 & 901 & 732 & $-18,8$ \\
\hline Thailand & 206 & 167 & -39 & 1322 & 955 & $-27,8$ \\
\hline China & 181 & -64 & -245 & 2061 & 1911 & $-7,3$ \\
\hline Indien & 139 & 76 & -63 & 567 & 620 & 9,3 \\
\hline Indonesien & 156 & 36 & -120 & 627 & 908 & 44,8 \\
\hline Libanon & 12 & 17 & 5 & 127 & 0 & $-100,0$ \\
\hline Pakistan & 42 & 18 & -24 & 236 & 260 & 10,2 \\
\hline Saudi-Arabien & -6 & 7 & 13 & 224 & 184 & $-17,9$ \\
\hline Verein. Arab. Emirate & 55 & -51 & -106 & 327 & 416 & 27,2 \\
\hline Vietnam & -19 & -40 & -21 & 159 & 47 & $-70,4$ \\
\hline Mittel- und Südamerika & 6205 & -1236 & -7441 & 64903 & 67047 & 3,3 \\
\hline Argentinien & 533 & -542 & -1075 & 1701 & 654 & $-61,6$ \\
\hline Brasilien & -808 & -616 & 192 & 5636 & 3717 & $-34,0$ \\
\hline Chile & -163 & -8 & 155 & 831 & 436 & $-47,5$ \\
\hline Mexiko & 941 & 184 & -757 & 5033 & 4198 & $-16,6$ \\
\hline Costa Rica & 13 & 17 & 4 & 158 & 217 & 37,3 \\
\hline Ecuador & 55 & 42 & -13 & 439 & 453 & 3,2 \\
\hline Guatemala & -8 & -8 & 0 & 80 & 133 & 66,3 \\
\hline Kolumbien & -70 & -189 & -119 & 1151 & 1208 & 5,0 \\
\hline Peru & 36 & -42 & -78 & 291 & 262 & $-10,0$ \\
\hline Uruguay & 90 & 152 & 62 & 414 & 586 & 41,5 \\
\hline Venezuela & 176 & -239 & -415 & 1163 & 664 & $-42,9$ \\
\hline Offshore-Finanzzentren & 5546 & -512 & -6058 & 46447 & 49808 & 7,2 \\
\hline Afrika & 143 & -491 & -634 & 4403 & 3496 & $-20,6$ \\
\hline Ägypten & 82 & 48 & -34 & 602 & 413 & $-31,4$ \\
\hline Elfenbeinküste & -6 & -93 & -87 & 117 & 61 & $-47,9$ \\
\hline Marokko & 37 & 25 & -12 & 288 & 210 & $-27,1$ \\
\hline Nigeria & 8 & 3 & -5 & 31 & 34 & 9,7 \\
\hline Südafrika & 54 & -238 & -292 & 1246 & 1252 & 0,5 \\
\hline Welt insgesamt & 30783 & 11811 & -18972 & 425281 & 409655 & $-3,7$ \\
\hline
\end{tabular}

Quelle: Schweizerische Nationalbank, Das schweizerische Bankwesen 2002, 2003. 


\subsection{Personal in den schweizerischen Unternehmen im Ausland \\ B. Personalbestand am Jahresende}

Zahl der Beschäftigten

Land

$20012002 \quad \begin{aligned} & \text { Veränder. } \\ & 02 / 01 \text { in } \%\end{aligned}$

Industrieländer, MOEL/NUS

und andere europ. Länder ${ }^{1}$

1268384

2002

$02 / 01$ in $\%$

Nichteurop. Entwicklungsländer

und fortgeschrittene Entwicklungsländer

Asien $^{2}$

Lateinamerika

456394

1341145

5,7

Afrika

230882

485595

161776

254290

6,4

Aufteilung nach Ländern :

Europäische Union

63736

160106

10,1

Belgien

767683

71199

$-1,0$

Dänemark

23107

Deutschland

14766

Finnland

215655

Frankreich

15668

800693

11,7

Griechenland

Italien

136930

24534

4,3

Luxemburg

8844

6075

13204

6,2

Niederlande

\begin{tabular}{ll}
\hline Österreich & 348 \\
\hline Portugal & 102
\end{tabular}

\begin{tabular}{l} 
Portugal \\
\hline Schweden \\
\hline Spanien
\end{tabular}

Grossbritannien $^{3}$

64568

2379

225573

15891

$-10,6$

Übriges Europa

27860

34870

10235

28292

58830

149003

4,6

Ubriges Europa

Kroatien

\begin{tabular}{l}
\hline Polen \\
\hline Russische Föderation \\
\hline Tschechische Republik \\
\hline Slowakei \\
\hline Ungarn \\
\hline Türkei
\end{tabular}

\section{USA, Kanada}

119606

111525

13238

3237

19944

12311

19148

9211

5359

Andere nichteuropäische entwickelte Länder

Australien, Neuseeland

Japan

3393

11735

9266

339827

58615

29271

29344

63886

2499

1,4

1,4
8,8

638


Zahl der Beschäftigten

\begin{tabular}{|c|c|c|c|}
\hline \multirow{2}{*}{ Land } & \\
\hline & 2001 & 2002 & $\begin{array}{l}\text { Veränder. } \\
02 / 01 \text { in \% }\end{array}$ \\
\hline Asien & 221616 & 244516 & 10,3 \\
\hline Hongkong & 14901 & 16553 & 11,1 \\
\hline Südkorea & 5327 & 6227 & 16,9 \\
\hline Malaysia & 18587 & 21011 & 13,0 \\
\hline Philippinen & 13027 & 13297 & 2,1 \\
\hline Singapur & 18898 & 19298 & 2,1 \\
\hline Taiwan & 10520 & 10197 & $-3,1$ \\
\hline Thailand & 36962 & 36137 & $-2,2$ \\
\hline China & 40496 & 54514 & 34,6 \\
\hline Indien & 20274 & 20979 & 3,5 \\
\hline Indonesien & 10834 & 13444 & 24,1 \\
\hline Libanon & 619 & 743 & 20,0 \\
\hline Pakistan & 5159 & 5271 & 2,2 \\
\hline Saudi-Arabien & 3490 & 3669 & 5,1 \\
\hline Verein. Arab. Emirate & 1301 & 1829 & 40,6 \\
\hline Vietnam & 5678 & 6159 & 8,5 \\
\hline Mittel- und Südamerika & 161776 & 160106 & $-1,0$ \\
\hline Argentinien & 12406 & 12883 & 3,8 \\
\hline Brasilien & 70019 & 70698 & 1,0 \\
\hline Chile & 9366 & 8800 & $-6,0$ \\
\hline Mexiko & 26453 & 26300 & $-0,6$ \\
\hline Costa Rica & 2361 & 2200 & $-6,8$ \\
\hline Ecuador & 3842 & 4118 & 7,2 \\
\hline Guatemala & 1438 & 1613 & 12,2 \\
\hline Kolumbien & 9129 & 6793 & $-25,6$ \\
\hline Peru & 4118 & 4680 & 13,6 \\
\hline Uruguay & 924 & 872 & $-5,6$ \\
\hline Venezuela & 8869 & 8111 & $-8,5$ \\
\hline Offshore-Finanzzentren & 7566 & 6529 & $-13,7$ \\
\hline Afrika & 63736 & 71199 & 11,7 \\
\hline Ägypten & 8290 & 8027 & $-3,2$ \\
\hline Elfenbeinküste & 2424 & 2263 & $-6,6$ \\
\hline Marokko & 3201 & 3310 & 3,4 \\
\hline Nigeria & 3938 & 3422 & $-13,1$ \\
\hline Südafrika & 21331 & 25909 & 21,5 \\
\hline Welt insgesamt & 1724778 & 1826740 & 5,9 \\
\hline
\end{tabular}




\section{Anmerkungen zu den Tabellen 2.4.A. und 2.4.B.}

\section{Grunddefinition :}

Wer eine Direktinvestition tätigt, will einen direkten und dauerhaften Einfluss auf die Geschäftstätigkeit eines Unternehmens ausüben (indem er eine Tochtergesellschaft oder eine Filiale im Ausland gründet oder sich mit mindestens $10 \%$ am Kapital eines Unternehmens im Ausland beteiligt).

\section{Datenerhebungsmethode :}

Die Schweizerische Nationalbank erhebt jährlich die Daten über die schweizerischen Direktinvestitionen im Ausland. Die Erhebung ist obligatorisch für alle Unternehmen mit einem Direktinvestitionskapital von über 10 Millionen Franken.

Die Unternehmen geben Auskunft:

๖ über die Finanzbewegungen auf dem Beteiligungskapital (Gründung von Tochtergesellschaften, Erwerb, Verkauf, Kapitalerhöhungen). Das Minuszeichen (-) gibt eine Kapitalrückführung in die Schweiz an (Tabelle 2.4.A linke Spalte);

$\square$ über die am 31. Dezember investierten Kapitalbestände (Beteiligungskapital und Konzernkredite) (Tabelle 2.4.A rechte Spalte). Das Investitionsvolumen (Kapitalbestand) im Ausland am Ende eines Jahres entspricht nicht unbedingt dem Bestand des Vorjahres plus dem Fluss des Jahres, da die Bestandsänderungen sich auch aus Faktoren ergeben können, die nicht in den Kapitalflüssen erscheinen (Erwerbungen im Ausland, finanziert durch die reinvestierten Gewinne von Tochtergesellschaften im Ausland, Entwicklung der Wechselkurse, Änderungen der Buchhaltungsmethoden, usw.);

$\checkmark$ über ihren Personalbestand in den Tochtergesellschaften und Filialen im Ausland (Tabelle 2.4.B).

\section{Aufteilung der Investitionen nach Ländern :}

Das in den Tabellen aufgeführte Land ist soweit wie möglich das Land des zuletzt Begünstigten (last beneficial owner) der ausländischen Direktinvestitionen der Schweiz. Dieses Prinzip kann jedoch nicht immer angewendet werden (zum Beispiel im Fall der Karibikstaaten, die Drehscheiben internationaler Kapitalbewegungen sind, oder im Fall der Steuerparadiese).

Siehe auch die Tabellen 4.1 und 4.2 betreffend die Investitionen nach der Klassifikation des Entwicklungshilfeausschusses DAC (Liste der Entwicklungs- und Transitionsländer am Schluss des Jahrbuchs).

Der Gesamtbetrag der Direktinvestitionsflüsse belief sich im Jahr 2002 auf 11,8 Milliarden Franken. Gemäss den Tabellen im 4. Teil der Statistiken wurden 1,9 Milliarden Franken in den Entwicklungsländern investiert (davon 1,4 Milliarden in Slowenien). 1,7 Milliarden Franken wurden in den fortgeschritteneren Entwicklungsländern und 0,3 Milliarden in den MOEL/NUS investiert. 
